\title{
Mit Pflanzenkräften aus dem Stimmungstief
}

\author{
In der Behandlung von Depressionen hat die Phytotherapie, insbeson- \\ dere der Einsatz von Johanniskraut, mittlerweile einen festen Platz. \\ Was bei der Verordnung zu beachten ist, erläutert Prof. Beer in diesem \\ Beitrag.
}

\section{? Bei welchen Arten von Depressionen kann man mit Phytotherapeutika hel- fen? Welche Phytopharmaka kom- men in Frage?}

\section{Als Alternative zu synthetischen An-} tidepressiva steht als pflanzliche Droge Johanniskraut zur Verfügung. Indikationen für Johanniskrautextrakt sind die leichte, vorübergehende depressive Störung, mittelschwere depressive Episoden und die psychovegetativen Störungen sowie depressive Verstimmungszustände, Angst und/oder nervöse Unruhe. Von der Europäischen ArzneimittelAgentur liegen ein sog. „well establised use“ und ein "traditional use“ vor. Die pharmakologischen und klinischen Informationen zu Johanniskraut umfassen die Inhaltsstoffe, Wirkungen und den Wirkmechanismus, Daten zur Humanpharmakologie und klinischen Wirksamkeit, zur Dosierung, zu Gegenanzeigen sowie zu Wechsel- und Nebenwirkungen.

Die Inhaltsstoffe wie $0,1-0,3 \% \mathrm{Ge}$ samthypericin (z. B. Hypericin, Pseudohypericin, Protohypericin), 2-4\% Phloroglucinderivate (z.B. Hyperforin), 2-4\% Flavonoide und Gerbstoffe hemmen die Serotonin-, Noradrenalin-, Dopaminwiederaufnahme, bewirken eine Hemmung an GABA-A-, GABA-B- und glutaminergen Rezeptoren.

In zahlreichen kontrollierten Studien gegenüber Placebo bzw. verschiedenen synthetischen Antidepressiva und Cochrane-Analysen wurden klinische Wirksamkeit und Gleichwertigkeit von Johanniskrautextrakten belegt.

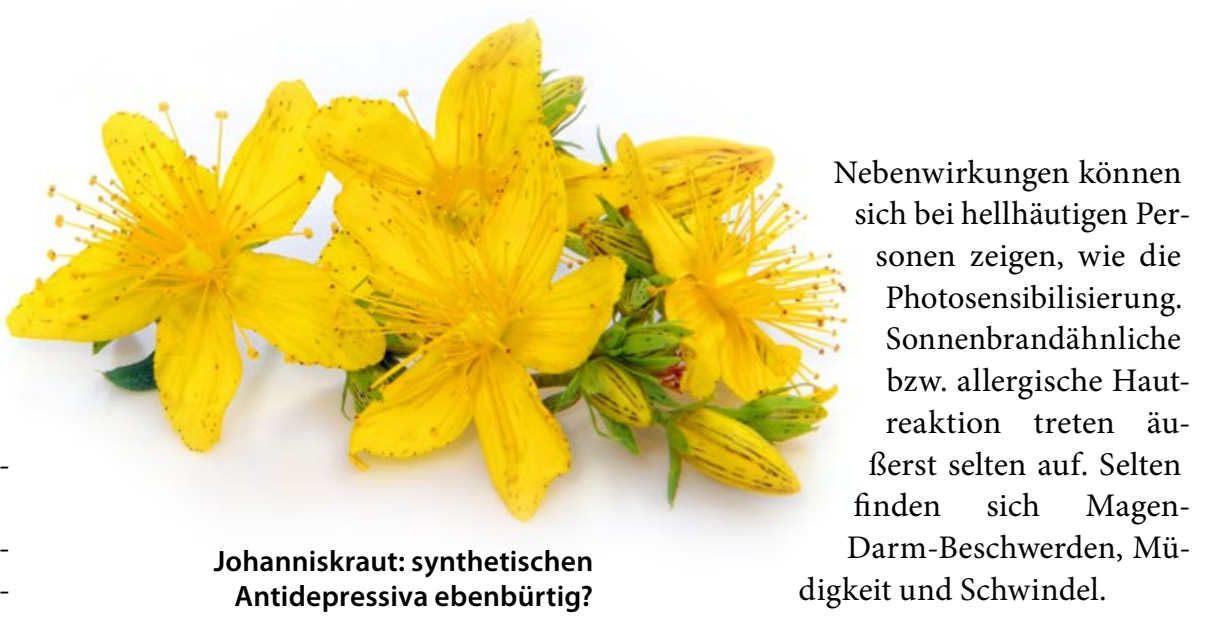

\section{? Wann darf Johanniskraut nicht ein- gesetzt werden?}

Als Gegenanzeigen für die Einnahme gelten die Lichtempfindlichkeit, schwere depressive Episoden, gleichzeitige Einnahme von Cyclosporin, Tacrolimus, Indinavir u. a. Proteasen-Hemmstoffe in der HIV-Behandlung, Irinotectan, Imatinib u. a. Zytostatika, Antidepressiva und Kinder unter 12 Jahren.

\section{Welche Interaktionen sind zu be- achten?}

Wechselwirkungen bestehen mit CYP3A4, 2C9, 2C19 und P-gp. Zur Wirkungsabschwächung kann es bei Antikoagulanzien, Ciclosporin, Tacrolismus, Digoxin, Indinavir und anderen Protease-Hemmstoffen in der HIV-Behandlung, Irinotecan, Imatinib, Zytostatika, Amitriptylin, Nortriptylin, Midazolam, Theophyllin, zur Wirkungsverstärkung vor allem bei Nefazodon, Paroxetin und Sertralin kommen.

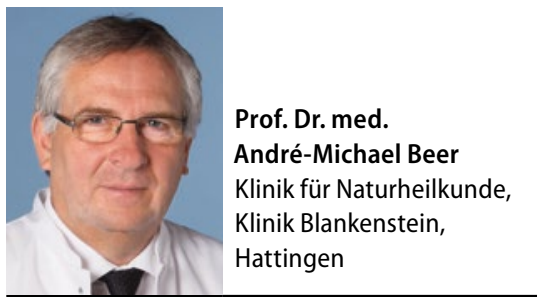

\section{Wann stößt die Phytotherapie bei der Behandlung von Depressionen an ihre Grenzen?}

Die Grenzen der Anwendung von Johanniskrautextrakten sind vor allem immer dann erreicht, wenn die Indikationen und Kontraindikationen, für die die Arzneimittel amtlich zugelassen sind, nicht beachtet werden

\section{?elche weiteren naturheilkundli- chen Maßnahmen sind zu empfehlen?}

Naturheilverfahren wirken am besten als sog. „Komplextherapie“. Dies bedeutet, dass neben dem Einsatz von Johanniskrautextrakten beispielsweise auch ordnungstherapeutische Gespräche, ein kalter Gesichtsguß nach Kneipp, stimmungsaufhellende Bauchwickel mit ätherischen Ölen, Hinweise auf ausreichend Bewegung im Alltag und eine ausgewogene Ernährung zum Behandlungsprogramm gehören. 